\title{
La saillance dans les campagnes publicitaires de communication sociale : analyse des ressources linguistiques et sémiotiques
}

\author{
Eurrutia Cavero, M. \\ Universidad de Murcia (Letras) \\ mercedes.eurrutia@um.es
}

\section{Introduction}

Selon F. Landragin la notion de saillance « est liée à l'émergence d'une figure sur un fond, que cette émergence soit motivée par des aspects physiques en référence à la perception de la parole ou du texte écrit ou par des aspects plus sémantiques voire cognitifs liés à la compréhension du langage » (2004:1). Prenant appui sur cette théorie, nous nous proposons de dégager, lors de la présente étude, les instruments dont se sert la communication sociale publicitaire pour marquer la saillance physique et la saillance cognitive; deux notions qui ne sont pas tributaires de la modalité linguistique ou visuelle à laquelle on les applique.

Partant d'un corpus de documents authentiques composé d'un échantillon de publicités françaises apparues entre 2008 et 2011, classées en fonction de la thématique abordée (accidents de la route, maladies à répercussion sociale et campagnes contre la consommation de substances dangereuses) et de la cible visée nous passerons en revue des ressources diverses servant à marquer la saillance (parmi d'autres celles qui font appel à la sémiologie, à la psychologie... ).

L'étude proposée nous permettra de tirer des conclusions sur la structure informationnelle sur laquelle la publicité sociale repose selon le contexte et la situation communicative envisagée et de mettre en évidence un fait incontestable : à la différence de la communication publicitaire commerciale dont le but essentiel est de favoriser la consommation, les campagnes publicitaires de communication sociale prônent des modifications de comportement ; on dirait qu'elles sont plutôt anti-consommatrices.

\section{Délimitation de la notion de saillance}

D'après Jouve « La communication publicitaire nous révèle l'homme, dans ses aspirations parfois les plus secrètes et notre société, dans ses valeurs mouvantes et éternelles » (Jouve, 2000 : 3). La publicité l'informe, elle est toujours présente dans sa vie sociale, professionnelle, voire sentimentale, remplissant en plus d'une fonction informative, une fonction de prescription, de positivation, et d'unification sociale.

Pour atteindre ses objectifs, la communication sociale en publicité se sert des phénomènes de saillance dont la description précise, exige la mobilisation de disciplines diverses : Sciences de l'homme et de la société en rapport avec la Linguistique et les Sciences de l'information et de la communication ; Sciences cognitives appliquées à la Linguistique et à la Psychologie... Néanmoins, une distinction s'impose entre deux notions de saillance : si la saillance physique est liée à la trace physique du message visuel ou linguistique, être saillant du point de vue cognitif, c'est « ressortir en premier de ses intentions de perception, de son attention visuelle et de sa mémoire à court terme, de ses expériences personnelles et de ses émotions » (Lits, 2008 : 28). La saillance cognitive fait donc référence aux processus cognitifs et aux spécificités du sujet; là la Psychosociologie, la Neurophychologie, la Psychologie expérimentale ou la Pyschanalyse constituent des disciplines indispensables pour comprendre, déterminer voire initier les choix et attitudes de l'individu face à la communication, à la consommation et à la vie sociale. À une époque où les valeurs sont en crise, la publicité sociale se substitue à la morale, aux lois socioculturelles. Elle véhicule, plus ou moins directement, les codes et les règles de vie à suivre ; elle propose des 
schémas comportementaux et des référents remplissant le rôle éducatif de la société. Malgré son but positif, cette publicité de prévention se caractérise par un contenu essentiellement menaçant qui contextualise des réalités sociales volontairement refoulées. Son but: lutter contre les maux présents dans toute société et promouvoir les valeurs collectives nécessaires ; pour ce faire elle nous confronte à une réalité déplaisante, à des images violentes qui nous bouleversent. Et dans cette optique, la saillance physique, visuelle et linguistique, constitue le point de départ pour tout message linguistique. Cependant une précision s'impose : la notion de saillance linguistique, plus tardive, n'est pas l'objet aujourd'hui d'un consensus généralisé. Ce concept a considérablement évolué au cours de dernières années. Si Stevenson (2002) distinguait les aspects formels (prosodiques, lexicaux, grammaticaux de l'énoncé) des aspects sémantiques, plus récemment les études de Landragin (2004), s'appuyant sur les idées déjà exposées par Wolters (2001), développent une notion de saillance plus ample que nous adopterons au cours de la présente étude : la saillance constitue plutôt une tendance, un facteur indispensable dans la modélisation du sens dès une double perspective : production et/ou interprétation du message.

Comme Pattabhirama et Cercone (1990) nous pensons que la saillance (extérieure au sujet) et la pertinence (intérieure au sujet en fonction de ses buts et de ses motivations) sont strictement liées.

La saillance intervient au niveau des processus cognitifs inférieurs tels que l'intention (volonté du sujet de comprendre le message transmis), l'attention (façon dont un sujet focalise ses capacités de lecture ou d'audition), la mémoire à court terme (en étroite relation avec l'activation, l'accessibilité, la familiarité immédiate, la récence...), la mémoire à long terme (l'individu apprend le sens des mots de sa lange à travers des expériences qui lui sont propres et qui connotent des sens particuliers au sein de sa culture et de sa société), la personnalité du destinataire du message et sa situation immédiate (coordonnées spatiales, problèmes, affaires... ). Chaque sujet met en avant un certain élément du message en fonction de son affect et de ses émotions. La saillance concerne donc les mots, les entités, les propositions, les sons... qui sont dotés d'une signification particulière pour l'individu mais également les facteurs culturels car c'est c'est au sein de la société que l'individu élabore inconsciemment, son expérience culturelle singulière à nulle autre pareille. C'est dans ce sens qu'il faut parler de sujets culturellement saillants pour l'affect (par exemple, tout ce qui concerne la mort ou la souffrance) et comme le rappelle Rousselet et Fabre-Thorpe (2003), à propos de la saillance visuelle, le sexe.

Les mots, riches en connotations, se voient attribuer une saillance qui fait presque partie d'eux-mêmes, devenant déclencheurs automatiques d'émotions.

\section{La saillance dans les stratégies communicatives lors des campagnes de publicité sociale}

Dans le domaine de la publicité sociale, la situation de communication et l'interaction entre émetteur et destinataire sont irréductibles aux schémas généraux de la communication linguistique au sens strict. Ce sont les institutions, les organismes et les établissements publics ou privés, ceux qui s'occupent de faire des investissements économiques pour inculquer des comportements et des habitudes consommatrices favorables à la société (Ministère de la sécurité routière, association des droits des non fumeurs DNF...). Le discours publicitaire social repose donc sur un paradoxe caché : malgré les formes dialogiques strictes ou amples selon le cas, le cadre argumentatif est toujours maîtrisé par l'annonceur. Le lecteur est invité à prendre parti sur les faits, sur les déclarations... exposés dans la plupart des cas constatés par une réflexion en symbiose avec le message qu'on lui délivre. Il n'y a pas une manifestation interlocutive réversible : la participation du lecteur est restreinte au spectacle du monde souvent dysphorique qu'on met en scène.

Les affiches publicitaires sur lesquelles est basée la présente étude constituent un support chargé de transmettre un type de communication différée dans l'espace et dans le temps. Dans ces documents de nature informative et prescriptive les processus rétroactifs, illusoires ou sollicités, s'attachent aux prolongements cognitifs de l'acte publicitaire. Le public ciblé souvent restreint et dans la plupart des cas déjà intéressé à ce sujet, est invité à poursuivre l'interlocution par des procédés de différente nature qui 
prennent appui sur des supports linguistiques, iconiques, symboliques, sonores... La diversité de facteurs en jeu rend bien compte de l'indéniable complexité sémiotique et pragmatique de la communication publicitaire. En nous inspirant de la terminologie classique d'Austin (1970 : 137), nous considérerons trois dimensions des actes de discours publicitaires : dimension locutoire (production textuelle et iconique); dimension illocutoire (force de persuasion : visée descriptive, informative et argumentative, incitative) -ces deux dimensions portant sur la communication langagière- et dimension perlocutoire (effet de l'acte illocutoire, réactions du lecteur persuadé ou non par le message transmis).

Face à la communication publicitaire commerciale, globalement caractérisée par un acte illocutoire indirect, implicite, de nombreuses publicités sociales s'appuient sur des actes illocutoires explicitement constatifs à intention perlocutoire de type faire croire et par ce fait faire agir; ces actes sont donc implicitement directifs. On passe du savoir (je sais qu'il est mauvais de rouler trop vite), au vouloir (je devrais ne pas boire; je devrais porter la ceinture de sécurité en milieu rural et urbain) et surtout, à la volonté d'agir (je cesse de programmer des instructions et autres données dans le dispositif GPS après le départ...). La précision du contenu et de l'énoncé (techniquement convaincant et en rupture avec l'habituel quotidien) et les fréquentes références aux expériences personnelles, aux faits prouvés (relations entre tabagisme et cancer) et aux comportements "souhaités " des citoyens (respect du Code de la sécurité routière) sont essentiels pour inciter le sujet à l'action. La vérité du message transmis encourage le sujet à passer du croire au faire. Le discours publicitaire doit donc être assez crédible pour susciter la croyance et l'absence de toute réfutation de ce qu'il affirme. Cette croyance est rendue possible par la manipulation des désirs profonds des sujets (conscients / inconscients). Les énoncés constatatifs suscitant la valorisation (euphorie) ou dévalorisation (dysphorie) d'un événement ou d'un certain produit (ex. ceux qui nuisent à la santé), déterminent l'acceptation d'un conseil, d'une consigne à suivre, d'une norme à respecter, toujours dans un but précis : le bien-être, l'acceptation de l'individu au sein de la société à laquelle il appartient et de sa propre existence.

Le sujet passe éventuellement à l'action, conditionné par deux phases préalables : la phase cognitive et la phase mimétique. Le désir d'identification part dans la plupart des cas d'une valorisation d'une attitude positive fondée sur des conséquences adverses préalablement constatées dues à un comportement contraire à ce qui a été prescrit par les normes sociales. Et c'est dans ce sens que l'on peut affirmer que le discours publicitaire social opère une sémantisation qui transforme le simple objet en objet de valeur. Le passage d'un rapport objectif au monde à un rapport symbolique est fréquemment représenté par les possibles conséquences catastrophiques d'un certain événement ou d'un hypothétique comportement adopté. La détermination de la stratégie communicative à suivre est indispensable avant le lancement d'une campagne de sensibilisation. Les concepteurs doivent tenir compte des paramètres tels que le contexte socioculturel, les besoins, les désirs, les craintes... pour relever les ressorts psychologiques et déterminer les points d'impact sur la cible; le recours à des effets de persuasion divers tels que la peur (accidents de la route) ou l'érotisme (santé publique et maladies à transmission sexuelle) ; l'impact du choix argumentaire ; l'unité du thème ; la précision du contenu et de l'énoncé ; les références directes aux expériences personnelles, aux faits prouvés et aux comportements «idéaux » des citoyens ; le ton du message et notamment, la gestion du temps (d'une part, l'époque de lancement d'une campagne d'information doit coïncider avec la période où les individus sont les plus réceptifs au message ; d'autre part, l'information doit se dérouler sur une période scientifiquement définie, sans interruption).

\section{Facteurs de saillance physique dans le domaine de la communication sociale publicitaire}

\subsection{Facteurs de saillance liés à la forme et au sens de l'énoncé}

En publicité la communication peut s'établir au niveau rationnel, objectif (dénotatif) ou au niveau symbolique, subjectif (connotation). Saillance linguistique et visuelle jouent un rôle complémentaire. Le traitement visuel d'un message linguistique ou d'une certaine image peuvent susciter des associations d'idées différentes mais la légende peut jouer des rôles divers : orienter le lecteur, falsifier en détournant 
l'image ou avoir une fonction explicative ; dans ce cas, le choix des termes est essentiel. Par la mise en relief d'un élément du message, l'attention du récepteur est focalisée vers cet élément privilégiant sa prise en compte, le sens que le concepteur a réellement l'intention de nous transmettre.

\subsubsection{Fonctions du langage et saillance}

En nous fondant sur la classification établie par Jakobson (1963 : 35) sur les fonctions du langage, nous remarquerons comment le discours de communication sociale a pour fonction d'informer, d'expliquer, d'argumenter (Il y a 1000 et une manières de communiquer. Au volant, c'est le clignotant). Cette argumentation persuasive ou incitative se compose de différents niveaux où divers actants interviennent. Perelman (1988) a proposé d'appeler persuasive, une argumentation qui ne prétend valoir que pour un auditoire particulier et d'appeler convaincante celle qui est censée obtenir l'adhésion en termes de persuasion et d'action, de conviction et d'intelligence. Dans les publicités touchant le domaine de la santé, par exemple, il est courant d'inviter le lecteur à rechercher les arguments autorisés d'un expert (médecin traitant, pharmacien...) ; le référent est souvent à l'honneur (fonction référentielle). Le discours a une fonction d'implication, dédoublée : 1. implication directe par un ton impératif, une apostrophe, une injonction, une prise à témoin ; 2. implication indirecte soit par une volonté de s'adapter au récepteur, d'être persuasif, accessible (d'où un ton souvent pédagogique), soit par des formules qui prouvent qu'on s'est préoccupé de ses besoins (on le connaît), de ses désirs (on devine ses préférences). C'est par la mise en avant de certains éléments linguistiques (structures qui marquent l'obligation, emploi réitératif de l'impératif, de l'infinitif à valeur impérative...) qu'on le fait entrer en scène, qu'on le transforme en interlocuteur, en responsable de ses éventuelles imprudences.

Lorsqu'on analyse les affiches publicitaires on remarque aisément qu'à la différence de l'éventuelle consommation volontaire de spots publicitaires télévisuels, le destinataire d'une publicité parue dans un journal doit être interpellé et convaincu instantanément de lire le message qu'on lui propose. De ces contraintes spécifiques découle le fait que cette structure communicative axée d'abord sur la recherche de l'établissement d'un contact, est un système avant tout phatique (Jakobson, 1963: 217). La fonction phatique accompagne donc la fonction conative puisqu'il y a prise en considération du récepteur. Il tire une conclusion, une morale de l'histoire tout en restant disponible pour plus de renseignements. On passe alors de l'éducation à la prescription. Exemple : campagne contre le tabagisme passif : interdiction de fumer dans les lieux publics :
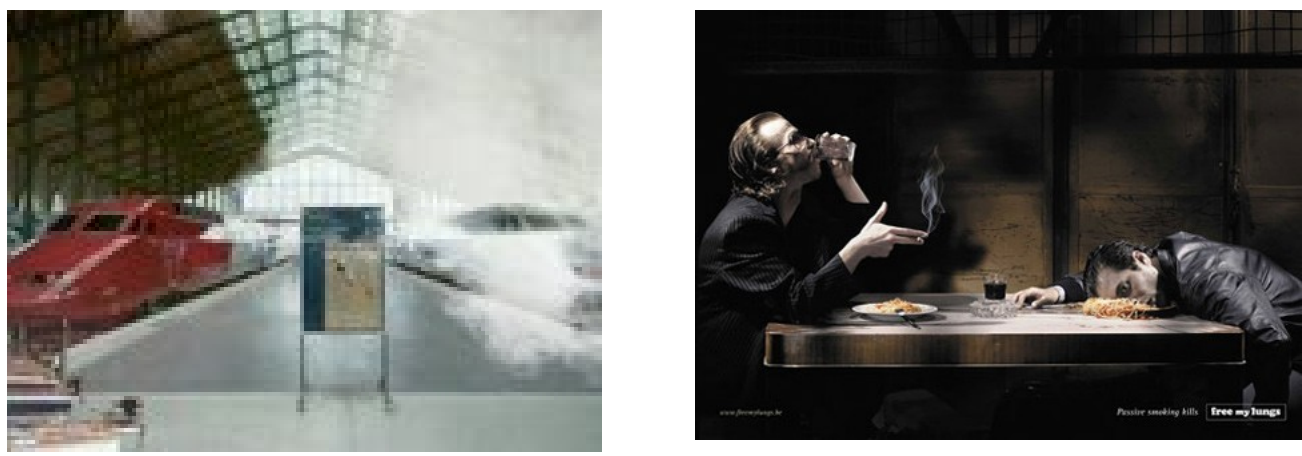

Le concepteur de la campagne publicitaire joue souvent sur l'émotivité et la sensibilité (fonction expressive ou émotive) du récepteur qui se sent implicitement ou explicitement impliqué dans une situation donnée. L'objectif recherché est ici d'établir le contact pour le contact (affectif, par exemple) et de le garder. En ce qui concerne la fonction poétique, elle se met en évidence lorsqu'on élève le discours rationnel au rang métaphorique, par exemple pour accentuer la violence des images. Deux exemples de 
publicité de choc pour les campagnes anti-tabac servent à illustrer cette constatation : 1. La cigarette casse ; 2. La cigarette tue : revolver ; à la place des balles, des cigarettes (message : fumer, tue).
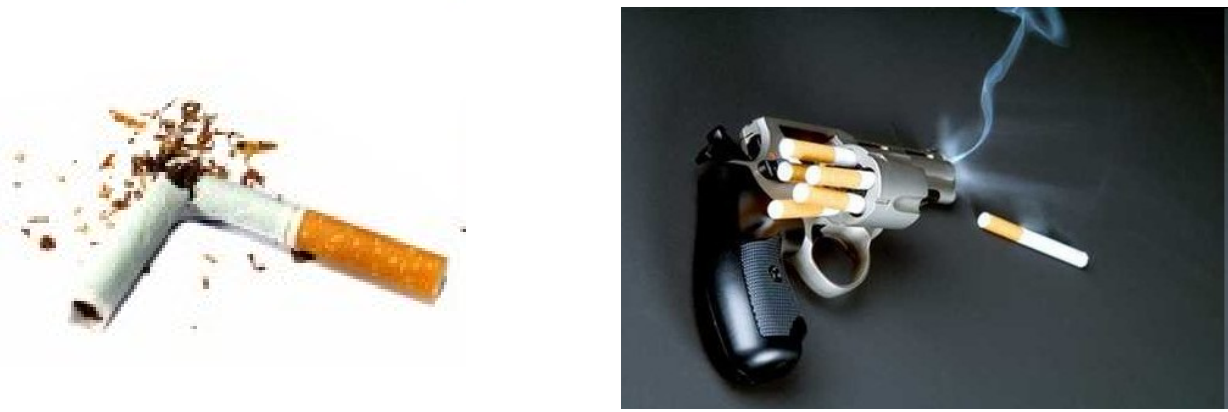

\subsubsection{Effets de rhétorique et de style qui marquent la saillance}

Une entité de discours devient tout particulièrement saillante par la combinaison de critères divers et l'emploi des techniques de la rhétorique jouant sur la grammaire et / ou sur la sémantique des mots ou de l'énoncé : altération de l'ordre habituel d'apparition des mots, mises en apposition, détachements en tête de phrase, réduplications, sémantique verbale, constructions clivées, constructions syntaxiques renforcées, accent de focalisation... et tout cela en fonction du but recherché :

1. Impliquer le récepteur en le prenant à témoin (Alcool au volant, autotestez-vous) ; en l'invitant à réfléchir (Vous voyez, vous comprenez...), en le provoquant (Sans préservatif c'est avec le SIDA que vous faites l'amour. Protégez-vous), en faisant semblant de l'interroger ou en le laissant seul juge (Vous êtes prêt à quoi pour gagner une minute? Prêt à embrasser le pare-chocs de la voiture devant vous? Prêt à vous handicaper la vie? Sur l'autoroute, maintenez au moins deux secondes avec le véhicule qui vous précède), en lui imposant des conduites à tenir pour éviter des conséquences négatives non souhaitées (Refuser d'allumer les feux le jour, c'est oeuvrer contre la sécurité routière).

Le message transmis, soit par des moyens verbaux et / ou sémiotiques, implicites et / ou explicites, s'inscrit ici dans un mouvement d'interactions conflictuelles à plusieurs niveaux. Cette notion de conflit fait référence à une prise de conscience réflexive plus dialogique que l'idée de « rupture » qui implique la destruction de quelque chose pour reconstruire. On retrouve dans ces slogans le fait « d'agir sur le récepteur» propre à ce discours préventif auquel nous sommes confrontés et celui de « faire agir le récepteur contre sa volonté » dans sa confrontation explicite à ce discours.

2. Renforcer l'affirmation et l'appuyer convenablement rendant l'assertion incontestable (il faut dire, il faut bien reconnaître), soulignant la perfection et la réussite (inégalable, incomparable, accompli...), accentuant la répétition (Ce n'est pas en roulant vite qu'on arrive plus vite chez soi), jouant sur l'absolu et le définitif (irréversible, totalement, absolument...).

3. Enfoncer le message dans la tête du récepteur au moyen de la tautologie et de l'anaphore qui a pour double effet le renforcement et la symétrie (Celui qui conduit, c'est celui qui ne boit pas).

4. Faire agir l'interlocuteur mettant en avant le critère de récence, c'est-à-dire la proximité textuelle ou temporelle de la dernière mention. Selon ce principe évoqué par Krahmer et Theune (2002) les entités les 
plus saillantes sont celles mentionnées le plus récemment. Même si nous considérons l'intérêt de ce principe, à notre avis la saillance est loin de se réduire à cette notion.

5. Se jouer de la controverse au moyen de l'épitrope, de l'épanothosde, de la contre-objection ou prolepse (Vous allez me dire que...), de la prétérition (Toutes les inspirations que vous prenez pourront détruire votre futur - tabagisme).

6. Idéaliser ou émouvoir en se servant des figures de style telles que l'antithèse (antilogie, antinomie et oxymore), la périphrase et notamment de l'hyperbole (tabagisme et cancer : Rapide. Lente. La cigarette aussi dangereuse que la balle mais plus vicieuse), l'opposition de deux mots, de deux expressions (tabagisme et hypertension: Pas de fumée sans feu; tabagisme et maladies cardiovasculaires : Cigarettes légères, risques lourds) :
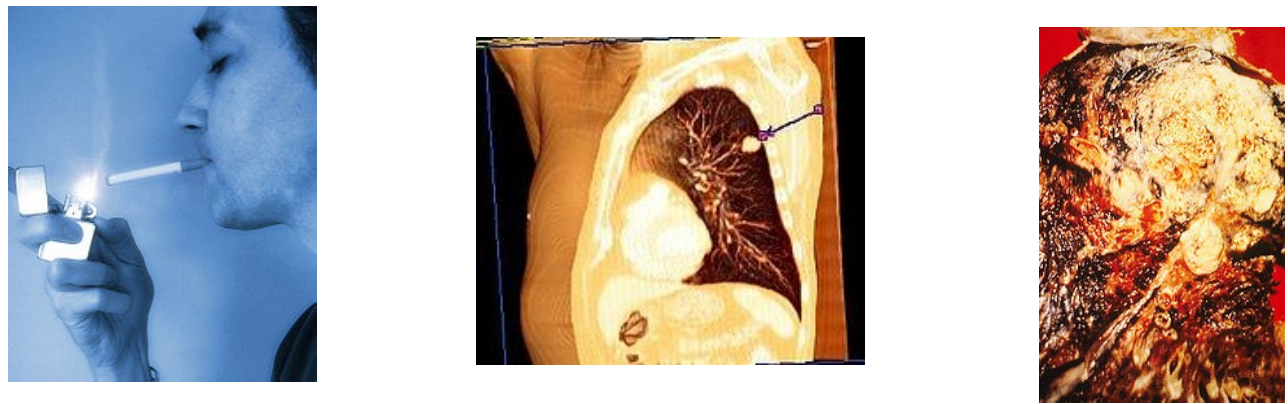

Opposition de deux pensées (accidents de la route : Vous êtes juste passé à l'orange, il passera juste sa vie à l'hôpital; un comportement incorrect facile à éviter peut entraîner des conséquences catastrophiques) :

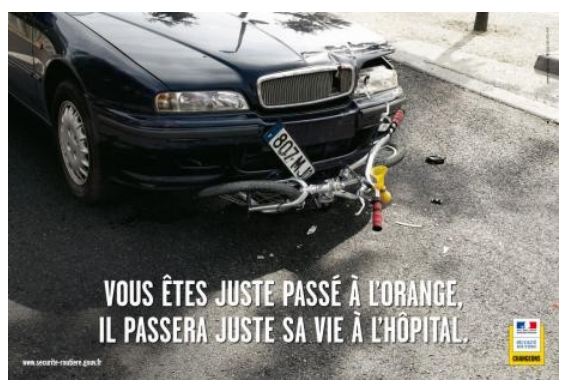

Juxtaposition de deux mots de sens identiques ou très voisins ; polysyndète ou synonymie reposant sur l'association d'idées: Attacher sa ceinture, c'est attacher sa vie (ceinture, vie). Campagne pour le port de la ceinture de sécurité :

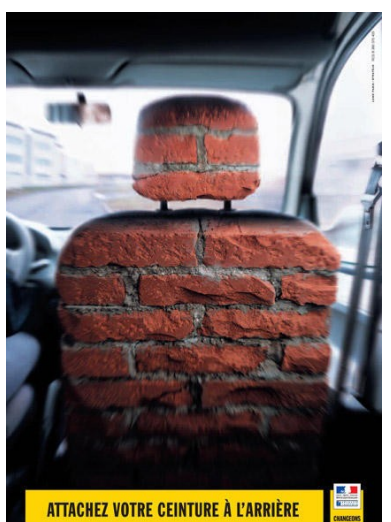


7. Suggérer de manière évocatrice par l'emploi de figures de style telles que la métaphore ou la métonymie, l'onomatopée, la litote, la synecdoque... Tabagisme : La belle au bois dormant, une mauvaise haleine qui n'attire pas le prince. L'association entre le tabac et les contes de fées constitue une opposition qui sert à amplifier le message :

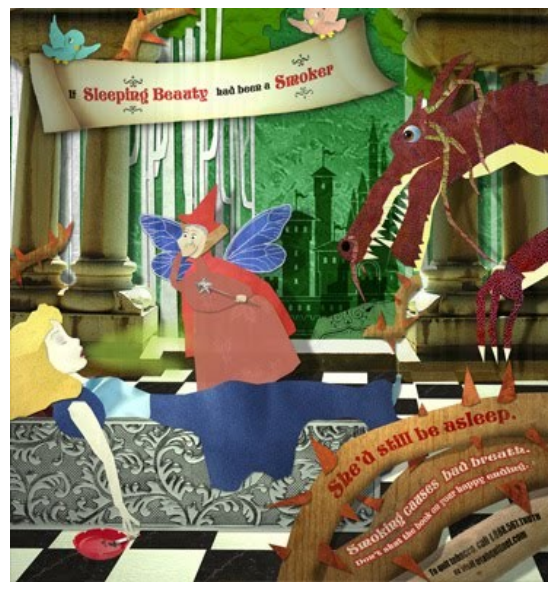

Accidents de la route: On peut sauver des vies sans pouvoirs exceptionnels; L'enchanteur Gayssot fustigeant les automobilistes irresponsables:

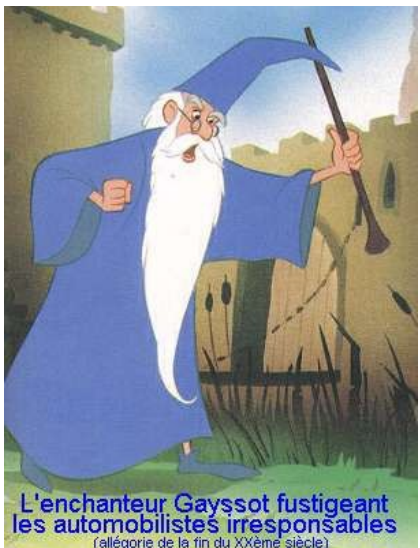


Ce dessin animé de Walt Disney (1960) a été inspiré du personnage légendaire, vu comme un magicien et un druide bénéfique qui commandait aux éléments naturels et aux animaux. Il est surtout mentionné dans la mythologie brittonique et dans le cycle arthurien dont il est l'un des personnages principaux.

Sida: le silence plombe l'ambiance. Ce slogan, choisi par l'association pour la Marche des Fiertés LGBT (lesbiennes, gays, bisexuels et transgenres) organisée en juin 2010 à Paris, joue sur le sens réel et figuré du verbe plomber, garnir de plomb et dans un sens familier, avoir un effet négatif sur, compromettre (Robert, 2004 : 1960). On souhaite ainsi interpeller la communauté LGBT sur son silence face au sida et sur les difficultés que rencontrent les séropositifs pour enjoindre à davantage de combativité et de solidarité.

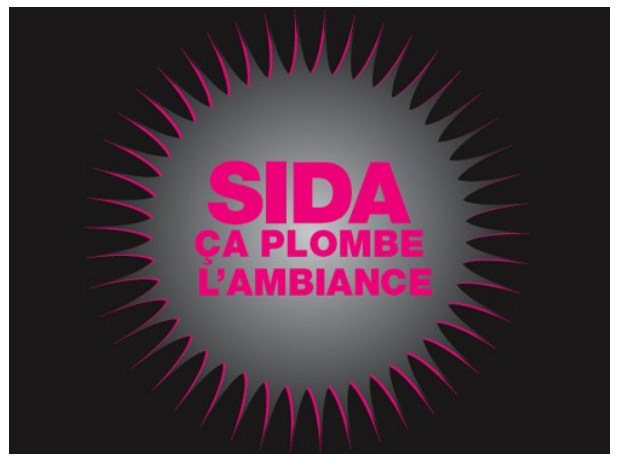

Ce slogan adopte des modalités diverses : Sida : ça plombe l'ambiance ou encore une autre plus chargée sémantiquement : Sida : ça plombe la vie. Avec les progrès thérapeutique, le sida tue moins vite mais il tue encore. Victimes de discriminations dans leur entourage, dans leur travail, dans leur vie quotidienne, les sidéens sont souvent isolés.

On peut suggérer de façon subjective en glissant insidieusement un mot dans un propos en apparence objectif ou en choisissant un terme qui portera en lui une connotation positive ou négative (Le samedi soir, éclatez-vous sur la piste de danse, pas sur le goudron) :

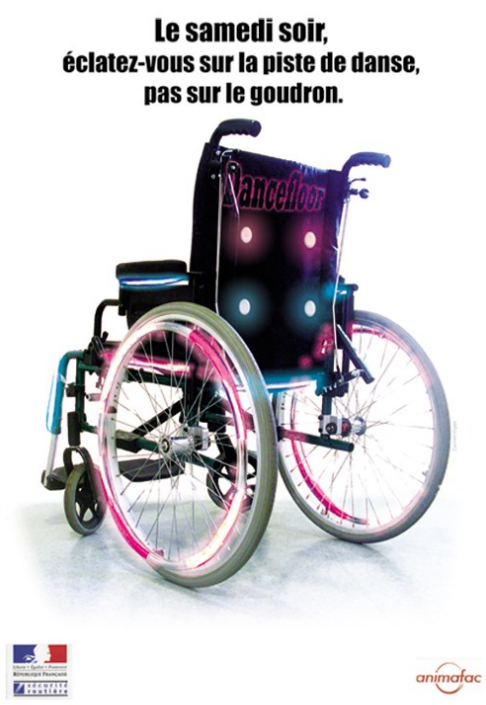


Les deux sens de base du verbe éclater sont mis en exergue; dans son usage non pronominal, éclater signifie «exploser, casser, faire voler en éclats » mais dans sa construction pronominale réfléchie, s'éclater devient synonyme "d'éprouver un violent plaisir dans une certaine activité », de " jouir» (Robert, $2004: 825$ ) ; le transfert sémantique opéré a pour effet un impact incontestable sur le locuteur. La violence des mots, très chargés sémantiquement et riches en connotations tragiques, contraste avec la simplicité des signes iconiques, susceptibles d'émouvoir les récepteurs. La saillance linguistique garde ici une analogie rigoureuse avec la saillance visuelle. Dans la scène la représentation graphique du fauteuil roulant se détache sur un fond blanc qui représente le néant. Cette représentation graphique du fauteuil roulant et le fauteuil lui-même en tant que référent, sont comparables à la scène verbale construite par le discours dans le sens de Victorri \& Fuchs (1996) et selon les principes des grammaires cognitives : Les imprudences au volant coûtent chères.

La saillance visuelle s'applique à cet objet mais elle tient compte à la fois des représentations graphiques, des propriétés attachées à celui-ci, de la configuration de la scène et surtout des états mentaux du sujet impliqué dans une pareille situation. De façon similaire, considérer que la saillance linguistique ne s'applique qu'aux seuls mots de l'énoncé serait une conceptualisation tout à fait réductrice. La structure informationnelle d'un énoncé doit partir donc de la détermination des entités du discours auxquels nous attachons une certaine saillance mais il faut également considérer les conclusions que nous pouvons tirer sur la structure du message et sur son sens.

On pourrait même constater un certain rapport entre propriété saillante et propriété prototypique dans cet objet qui fait partie de la vie quotidienne des handicapés dans la culture occidentale. On est conscient que comme l'a bien décrit Rastier (1991) à partir de la catégorisation des couleurs, la connaissance d'un prototype renfonce la saillance de certains objets mais alors que le prototype est lié à une représentation mentale durable et culturelle, on reconnaît ici une imprudence éventuelle; on parle donc d'une situation déterminée qui a eu lieu dans un environnement concret et dans un intervalle de temps précis, c'est-à-dire de saillance.

8. Renforcer le propos : - Par la récupération des valeurs spirituelles, morales, culturelles, sociales (SIDA : Jean est séropositif, Olivier ne le sait pas. Jean non plus d'ailleurs. Se faire dépister c'est aussi protéger les autres. De par sa nature l'emploi d'un nom propre peut être considéré comme plus saillant qu'une préposition ou un adverbe. Rappelons que les mots sont des indices conduisant au sens, ce sens concourant à la saillance :

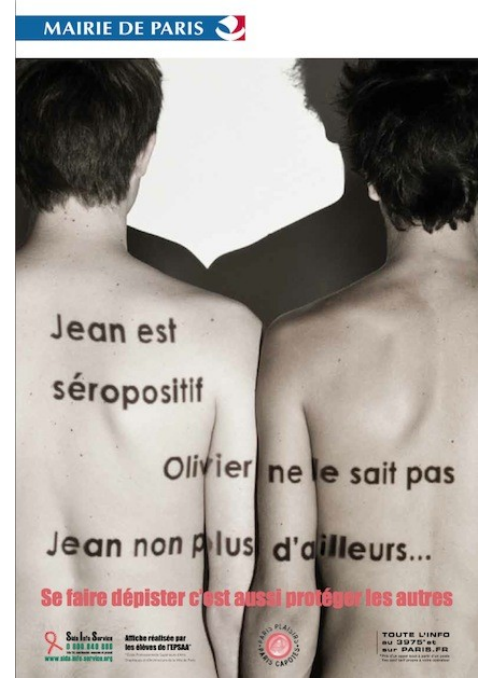


- Par des idées fortes et des termes de puissance (légende : Les accident de la route représentent la première cause de mortalité chez les 15-24 ans. Campagne contre l'excès de vitesse : Elle déchire ta caisse. (soulignons l'emploi du possessif en tant qu'effet de saillance) :

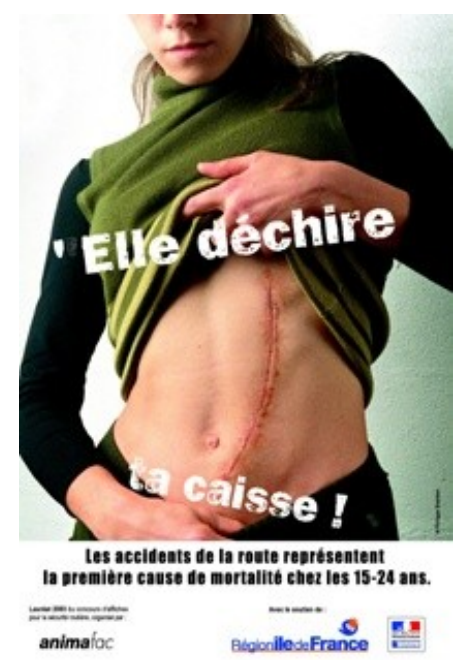

L'image de la jeune fille portant une cicatrice sur le torse évoque la polysémie du mot caisse : 1. caisse thoracique -le thorax se comporte comme une caisse de résonance-; 2. caisse, nom familier même argotique pour désigner la voiture (Robert, $2004: 330$ ). Les sens du mot caisse, les représentations construites à partir de ce mot gardent un rapport étroit avec les éléments iconiques de cet énoncé.

- Par une tonalité négative malgré l'idée positive transmise (gilet de sécurité : C'est jaune, c'est moche, ça ne va avec rien, mais ça peut vous sauvez la vie) :

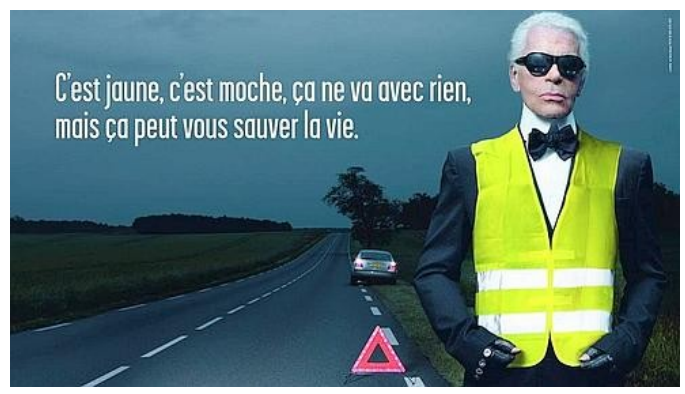

- Par l'emphase, l'exagération, les superlatifs, l'abondance d'épithètes...

- Par l'antiphrase ou le paradoxe -décalage entre le visuel et l'accroche : Parking payant, places disponibles au sous-sol (accidents de la route) : 


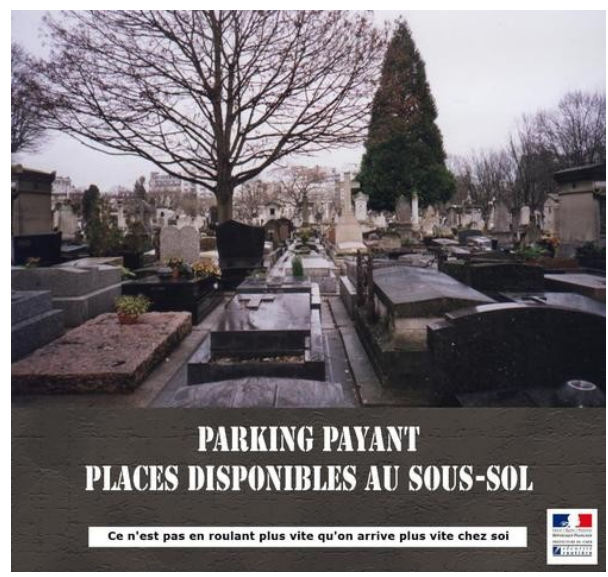

- Par les redondances et les répétitions de certaines structures de la phrase (Les trajets de tous les jours sont dangereux tous les jours) :

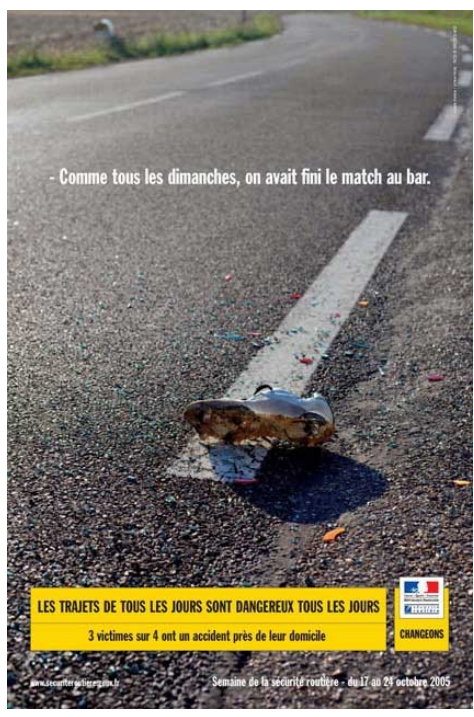

Ou par la répétition de certaines catégories grammaticales (Ça existe ça, les clignotants? alors, pourquoi vous ne les mettez pas?). Comme dans toute campagne de sensibilisation il est important de bien comprendre la portée de l'utilisation des clignotants. Lorsqu'on s'y habitue, ce geste anodin devient automatique et cela évite que le conducteur qui vous suit nous emplafonne l'arrière-train parce qu'il n'a pas compris que nous ralentissions. Ce slogan porte apparemment sur un rappel innécessaire : tout conducteur est capable d'identifier les clignotants mais pas tout le monde est disposé à les employer. Il s'agit plutôt d'une incitation à la réflexion sur une mauvaise conduite devenue systématique.

- Par un style dense, épique, lyrique, romanesque... (tabagisme : La belle au bois dormant, une mauvaise haleine qui n'attire pas le prince). 
9. Évoquer le système de valeurs par le chiasme. Ce croisement d'éléments dans une phrase ou un ensemble de phrases contribue à donner du rythme, à établir des parallèles. Le chiasme peut également souligner l'union de deux réalités ou renforcer une antithèse. Prenons le slogan publicitaire émis par un organisme d'assurances pour illustrer cette idée : Nous sommes au cœur de votre santé parce que votre santé nous tient à cœur. Le système de valeurs est ici «au cœur» de l'affaire par une structure dialogique comme montre l'emploi de votre (deux fois) et de nous. La thématique abordée " votre santé » est ici axiologisée. La compagnie d'assurances accorde la valeur la plus importante à la santé de chacun (tenir à cour), seule entité évoquée (votre santé individuelle). C'est pour cela (parce que) qu'elle essaie de se situer au cour de votre santé. On a l'impression d'une compénétration énonciateur / énonciataire sur cette base thématique. Une constatation se rend évidente : si les deux occurrences de santé ont la même signification, il n'en va pas de même des deux occurrences de cœur prises d'ailleurs dans des lexies plus larges (au cœur de, tenir à couur): la première renvoie à une localisation même abstraite (quelque chose qui regarde la précision, la justesse); la seconde, à une certaine évaluation (cette expression figurée signifie l'essentiel, le plus important).

10. Rétroagir poussé par la distance ironique du message publicitaire. Diverses publicités récentes prennent une allure plutôt interactive. Après l'installation d'espaces non-fumeurs dans les lieux publics ont paru en France diverses publicités faisant allusion à ce fait. Nous voulons insister tout particulièrement sur l'une d'elles que nous considérons comme très originale : l'annonce de Peter Stuyvensant. Sur une double page recto-verso on trouve ces mots : Espace fumeurs. Peter Stuyvesant avec un mode d'emploi inscrit sur le recto de la première page : Détachez cette page centrale. Tenez A de la main gauche, B de la main droite. Posez l'espace fumeurs ainsi obtenu sur votre tête et allumez-en une. La double page publicitaire se présente ici comme un adjuvant potentiel du lecteur, venu l'aider à satisfaire son besoin de fumer. Le lecteur de son côté, est supposé agir comme adjuvant du publiciste en déployant cette double page et en démultipliant ainsi ses possibilités de divulgation. Enfin, le publiciste se comporte en adjuvant du lecteur en lui fournissant des cigarettes accompagnées d'un possible et ironique équipement d'espace fumeur. Le montage complexe, interactif et asymétrique n'est là que pour proposer une façon de déjouer la loi anti-tabac et pour maintenir les ventes à défaut de les augmenter. Dans le cas le lecteur rétroagit sur le support publicitaire et non sur le produit même. Cette publicité atypique et étonnante nous surprend justement à cause de son excentricité.

L'ironie joue souvent sur l'antiphrase et le paradoxe comme montrent certaines campagnes publicitaires de sensibilisation sur le sida. Un slogan sert à illustrer l'idée exposée : Le sida est beau. Cet été protégezvous.

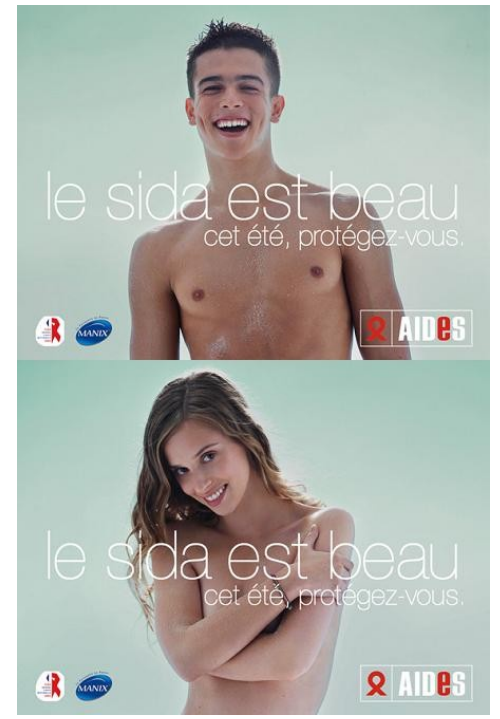


La politesse feinte et le ton ironique de cet énoncé frappent tout particulièrement. Le mots clés été, beau, protéger et SIDA entretiennent des rapports conflictuels au niveau sémantique. On a là un énonciateur simulé qui prend la parole et nous interpelle explicitement, en se mettant à la place de l'interlocuteur et en acceptant ses présupposés mais pour tenir un discours contraire à ce qu'il veut faire admettre. Cette inversion feinte des rôles est une structure rhétorique, proche de l'ironie, à savoir le paradoxe qui inverse les valeurs, le mal est retourné en bien (beau). Le raisonnement semble illogique et contraire aux données de l'expérience, et pourtant contient une vérité dérangeante et éclairante : Le SIDA est beau. Cet été protégez-vous. Ce slogan nous renvoie à un autre : Vous avez raison de ne pas vous protéger du SIDA, on vit très bien avec. L'antiphrase qui est utilisée ici consiste à dire l'inverse de ce qu'on veut laisser entendre pour amener l'interlocuteur à remettre de lui-même les choses en place. D'autant plus que celuici est doublement touché, car d'un côté, il est frappé par l'inconscience mise en œuvre dans le discours. Et de l'autre, il est impliqué explicitement par le déictique vous s'y reconnaissant peut-être ayant déjà pris des risques en ne se protégeant pas. L'image d'un beau jeune et son beau sourire a une évidente valeur métaphorique.

11. Mettre en relief un certain aspect du message en se fondant sur l'emploi restrictif des pronoms personnels. Les actants de différents niveaux de la publicité sociale sont désignés par des indices personnels (pronoms personnels et adjectifs possessifs), que ce soit sous la forme de déictiques qui renvoient directement à leurs référents extralinguistiques ou d'anaphoriques qui reprennent un élément du contexte. Deux tendances se détachent en ce qui concerne l'emploi des pronoms personnels : - un emploi restrictif des pronoms personnels qui situe au premier plan la première et la seconde personnes (du singulier et/ou du pluriel) ; locuteur et récepteur s'y trouvent directement impliqués ; - une prédilection pour l'indéfini on, expression la plus efficace de la référence personnelle. L'extension dénotative de ce pronom protéiforme doit être interprétée en fonction du contexte. Prenons l'exemple suivant d'une publicité des voitures $A u d i$ : «Un moteur de 280 chevaux, c'est émouvant. Un enfant qui traverse aussi. Pour tout constructeur automobile, il y a une évidente noblesse à concevoir un beau moteur [...] mais pour Audi, la noblesse se trouve aussi d'ailleurs, là où l'on n'oublie pas les réalités de la route, là où l'on prend conscience que le plaisir automobile ne se vit pleinement que maîtrisé [...] ». Dans cet énoncé on fait référence : dans le premier cas, au constructeur ou plutôt à tout constructeur qui se préoccupe de la sécurité de la voiture fabriquée ; dans le second cas, on dénote le public-cible, utilisateur potentiel de cette voiture. L'anonymat du fabricant et du public-cible coexistent dans un même champ référentiel en partageant un même but : la sécurité. Par l'attribution d'une valeur générale à cet énoncé on met en évidence un consensus collectif : le besoin de sécurité en circulation routière. Le pronom on contribue par ce fait à adoucir le circuit économique, soit en voilant qualitativement le fabricant du produit, soit en réunissant quantitativement ses utilisateurs dans une masse anonyme, soit en intégrant les uns et les autres dans un univers commun.

12. Importance de la catégorie sémantique du verbe comme facteur de saillance. Dans la description d'un événement, les conséquences de cet événement sont plus présentes dans l'esprit de l'interlocuteur que les conditions initiales. Si ces conséquences s'appliquent à l'agent, celui-ci en devient plus saillant que le patient; par contre, lorsque les conséquences s'appliquent au public ciblé, celui-ci devient plus saillant. De façon similaire, comme le montre Pearson (2001), dans les verbes de transfert, le receveur est plus saillant que le donneur et que l'objet transféré.

\subsection{Importance des signes iconiques dans les effets de saillance : orientation de l'image}

Comme on l'a bien constaté lors des paragraphes précédents la saillance linguistique est liée à la façon dont on présente ou traite le message. Elle concerne à la fois le lexique, la syntaxe, la sémantique, la pragmatique... Cependant la saillance physique est liée à la trace physique du message visuel, en entendant par là non seulement la présentation du message linguistique mais aussi les ressources iconiques (nature et disposition d'objets dans une scène), éléments indispensables de la structure informationnelle. Le processus de perception visuelle fait donc intervenir largement la notion de saillance. 
Notre attention s'arrête sur les éléments qui ressortent de notre environnement visuel jusqu'à axer les processus cognitifs sur ces seuls éléments. La saillance peut diriger la construction d'un message autour d'un élément déjà saillant ou peut au contraire, fournir un ensemble de moyens pour rendre saillant un autre élément et le porter de ce fait à l'attention du lecteur ou de l'interlocuteur.

Le développement des médias a suscité la multiplication des messages imagées ; les publicistes traduisent cette même tendance. À la différence de la publicité commerciale où l'image «représente dans le monde des valeurs ce qu'est le pseudo-événement dans le monde des faits : une sorte d'idéal factice fabriqué à grands frais » (Adam, 1997 : 196), dans le domaine de la publicité sociale, l'image reproduit vivement la réalité. Les sémiologues parlent aisément de ce "nouveau langage ». Mais comme le disait Umberto Eco «l'image ne comporte pas d'unités susceptibles d'être cataloguées » (1988:63); elle s'appuie donc sur un code faible qui doit être précisé par le langage (code fort). «Le slogan publicitaire -disait Roland Barthes appuyant cette idée- joue un rôle d'ancrage en forçant le récepteur à choisir l'une des multiples valeurs potentielles de l'image » (1964: 92).

Partant de ces considérations, nous nous proposons ici de réfléchir sur l'originalité et la force de l'argumentation iconique insistant sur les images employées avec une volonté délibérée de mettre en relief une valeur évocatrice ou symbolique ; on constante comme traits les plus saillants :

1. La violence explicite de certaines images. Selon Everaet-Desmedt « la publicité offre le spectacle d'un monde euphorique et d'un sujet positif » (1984: 133) ; cette affirmation valable dans le domaine de la publicité commerciale qui tend à la distanciation euphorique s'oppose à la conceptualisation de la publicité en communication sociale qui se caractérisent par privilégier la dysphorie (représentation de scènes d'attentats, de catastrophes, d'agonie...), la proximité du témoignage et des événements frappants de l'actualité. L'exposition du public à ces thématiques délicates déborde le cadre du seul discours informatif. Cette obligation de faire face à des situations conflictuelles qui s'inscrivent dans un contexte réel même quotidien constitue un acte menaçant, un acte qui met en évidence la notion de violence. L'effet positif de ces messages publicitaires est inférée des motifs négatifs suggérés, à partir de modèles de conduite négative à éviter. La parole dans cette sorte de communication sociale est contrainte d'opérer un déplacement de la perception en utilisant des moyens langagiers spécifiques et différents de ceux de la publicité commerciale et ajoutant plusieurs procédés d'implication explicite ou implicite à l'information pure et simple (prudence sur la route, non transgression des normes, appel à la solidarité, etc.). Dans sa dimension socioculturelle cette publicité préventive s'approprie le concept de violence qui se manifeste aussi bien au niveau verbal (énoncé textuel ou parlé ) qu'iconique.

Des exemples précis nous permettront d'illustrer ces notions :

\section{Campagnes de sensibilisation sur la sécurité routière.}

La fonction de ces campagnes est sans doute la lutte contre la transgression de normes. Ces recommandations adoptées par le Ministère de la Sécurité routière (le Ministère des transports), sont sensées veiller à l'application des règles permettant de circuler convenablement étant justifiées par des arguments appuyés sur des études scientifiques et/ou de tests effectués par des experts ou par " le comité d'experts » du CNSR (Comité National de la Sécurité Routière) dans une certaine région pendant une période préalablement déterminée et des résultats obtenus par l'application de cette mesure. La fonction référentielle devient dans ce cas élément essentiel de saillance. Après une phase d'expérimentation, nécessaire pour en faciliter l'acceptation sociale et pour tester le bien-fondé de la mesure acceptée, il faut bien délimiter les conditions indispensables à l'application d'une certaine mesure que plus tard, deviendra une obligation légale. Ces mesures doivent convaincre la politique du gouvernement et être approuvées par référendum (la Constitution Européenne). Cependant, des critiques sont fréquentes : la méconnaissance des spécificités liées à certains domaines voire le mépris de certains responsables par rapport au public ciblé. La parole dans cette sorte de communication sociale est contrainte d'opérer un déplacement de la perception en utilisant des moyens langagiers spécifiques et différents de ceux de la publicité commerciale et ajoutant plusieurs procédés d'implication explicite ou implicite à l'information pure et simple (prudence sur la route, appel à la solidarité, etc.). Dans sa dimension socioculturelle cette 
publicité préventive s'approprie le concept de violence qui se manifeste aussi bien au niveau verbal (énoncé textuel ou parlé) qu'iconique. L'irruption violente et incisive des scènes coexiste avec des messages, en principe éducatifs, qui partant souvent de présupposés négatifs tombent dans le cliché du mauvais goût. Les axes publicitaires sont bien déterminés:

\section{Campagnes publicitaires contre l'alcoolisme au volant.}

La lutte contre la consommation d'alcool au volant est une excellente initiative car l'alcoolisme est objectivement la cause d'une large proportion des accidents graves; au contraire de la vitesse qui n'est qu'un facteur aggravant. La Sécurité Routière a lancé cette campagne à l'approche des fêtes de fin d'année :

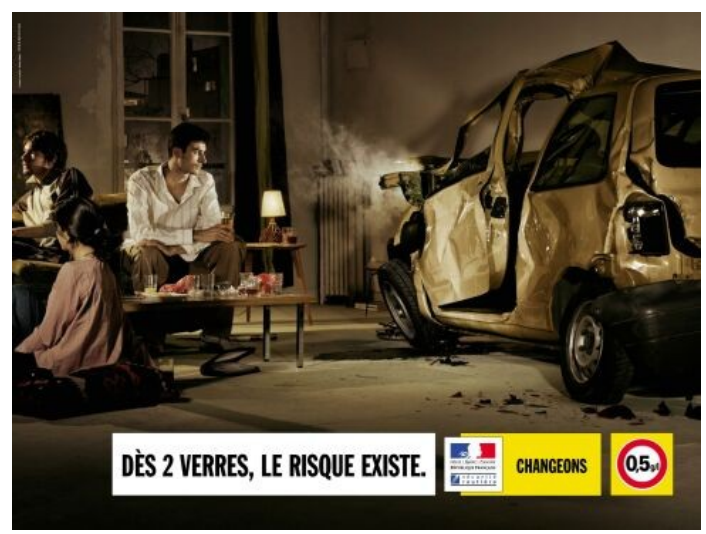

Cette mesure concernait tant les automobilistes que les motards :

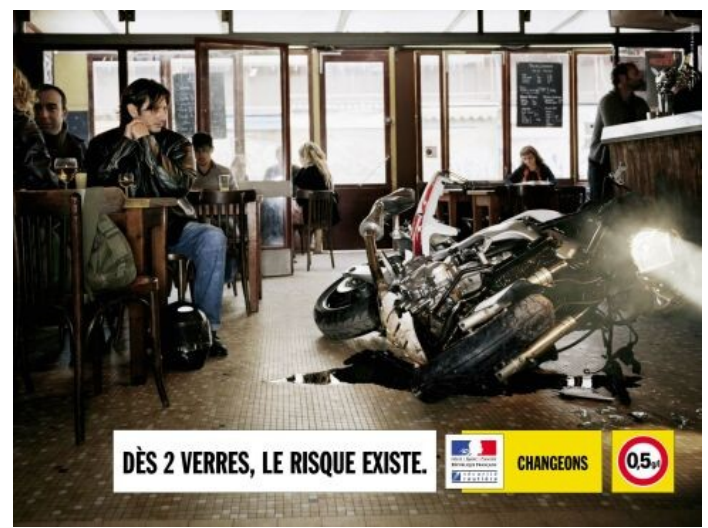

Le slogan Dès 2 verres le risque existe sous l'image d'un motard dans un bistrot qui a manifestement bu en excès et qui lance un regard contrarié à une moto cabossée, transmet un message assez ambigu. Si c'est sa moto, on ne voit pas bien le rapport avec le message de la prévention : il vient d'avoir un accident et il continue à boire. On dirait plutôt que le message incite le destinataire à boire. Les autorités de la Sécurité Routière voulant faire "un raccourci visuel» entre le moment où la prise de risque se fait (absorption d'alcool) et sa conséquence (accident grave dû à une alcoolémie positive) n'ont pas vraiment réussi à convaincre. En plus, le discours est faux dans un double sens : d'une part, la prise d'alcool est dangereuse dès la première gorgée car sous l'état euphorique le conducteur a l'impression de « maîtriser » 
son véhicule ; d'autre part, la borne virtuelle à $0,5 \mathrm{~g} / 1$ ne correspond à rien de précis médicalement parlant. Cette sorte de campagnes publicitaires montre bien que le compromis administratif existe même si les arguments invoqués sont souvent peu convaincants. D'après les critiques, ce discours est faux dans un double sens. Par défaut : la prise d'alcool est dangereuse dès la première gorgée (on a l'impression de «maîtriser » son véhicule, on se sent plutôt euphorique) et par excès : la borne virtuelle à $0,5 \mathrm{~g} / 1 \mathrm{ne}$ correspond à rien de précis médicalement parlant. Cette initiative d'instances officielles devrait être fondée sur une réelle justification.

Une constatation s'impose : de façon consciente ou inconsciente, les autorités peuvent inculquer aux citoyens des valeurs socioculturelles implicites. Le fait de cibler les motards dans une campagne de lutte contre l'alcoolisme renforce les connotations péjoratives fréquemment attribuées à ce secteur de la population souvent montré du doigt comme dangereux soit par les gouvernants soit par la société en général; malgré le sérieux des circonstances les créateurs des campagnes publicitaires ont recours à des effets de persuasion basés sur l'humour (en général, mal perçu dans ce domaine compte tenu des connotations graves des accidents) :
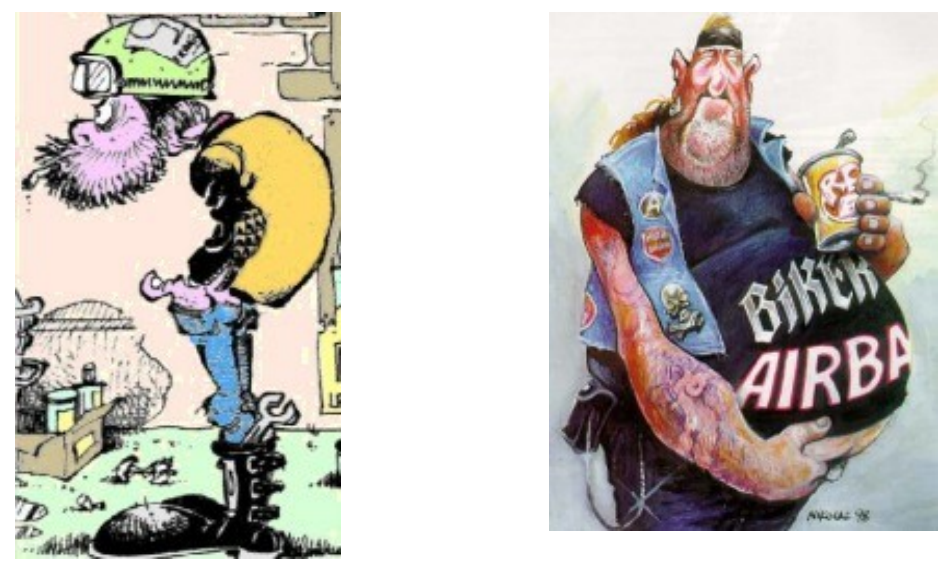

Campagnes contre l'excès de vitesse.
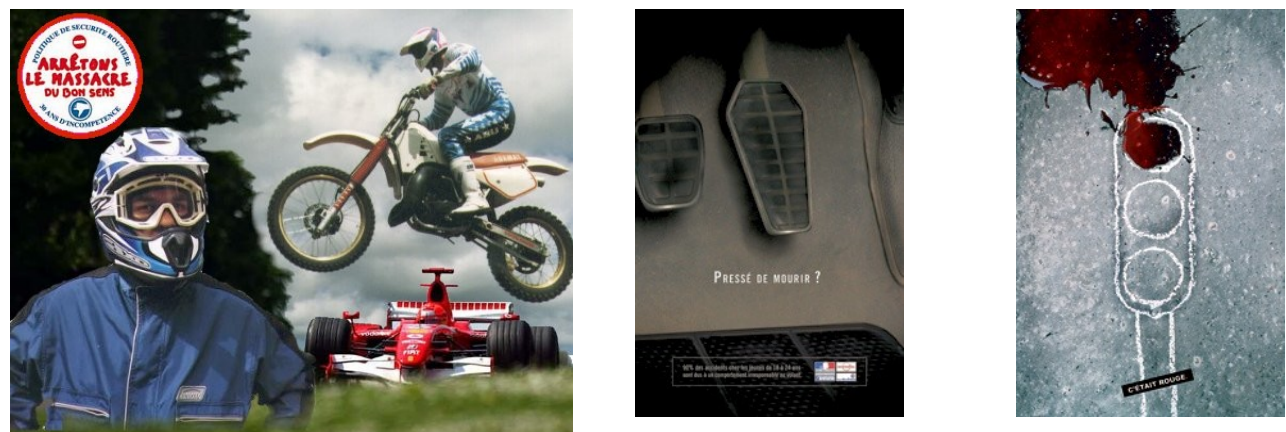

Campagnes de choc contre le téléphone au volant.

L'emploi du portable au volant a marqué notamment ces dernières années ; on se trouve joignable à tout moment et puis, on a la possibilité de « gagner du temps » (grand fantasme de notre monde occidental). 
On n'est pas conscient que téléphoner au volant multiplie le risque d'accident par 4 ou même par 6 dans les premières secondes (allongement du temps de réaction, rétrécissement du champ visuel, réduction instinctive et parfois brutale de la vitesse...) :
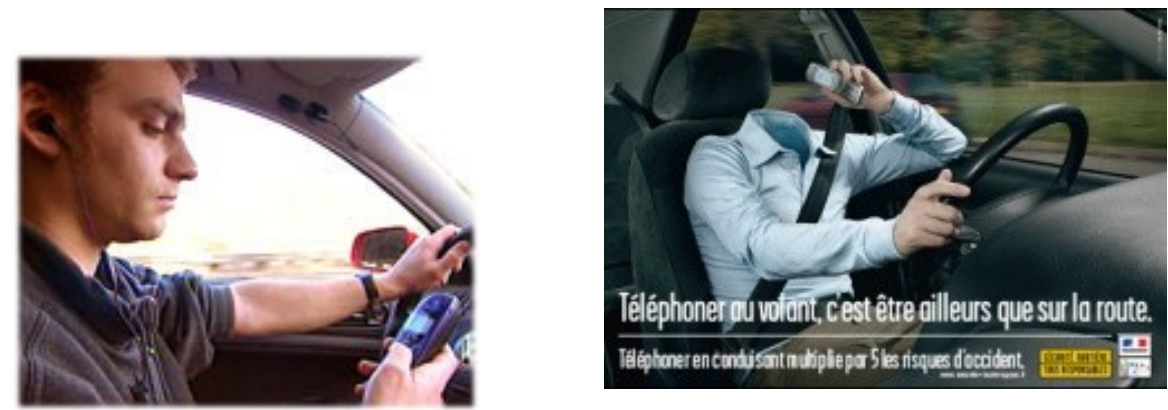

Citons la campagne conçue par l'agence publicitaire indienne Mudra Group dont le choix d'images sanglantes (le flou secoué, en arrière-plan, pour signifier le choc) est d'une violence qui dépasse amplement celle des campagnes anglaises ou françaises. Le slogan Ne lui parlez pas pendant qu'il / qu'elle conduit sort de l'ordinaire; il repose donc sur une remarque judicieuse : la notion de responsabilité partagée par les deux correspondants impliqués dans cet échange communicatif.
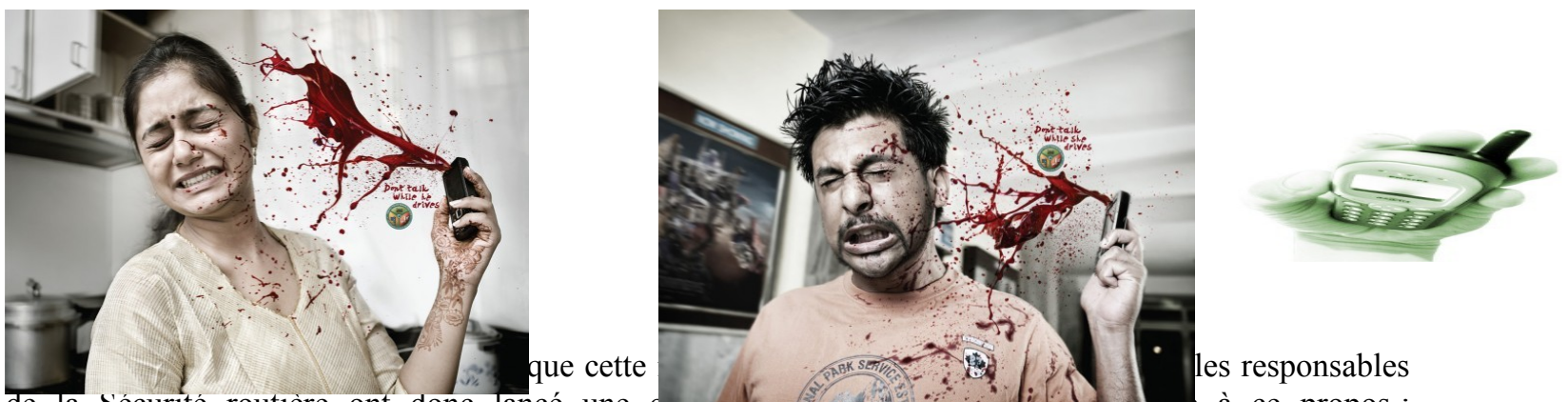

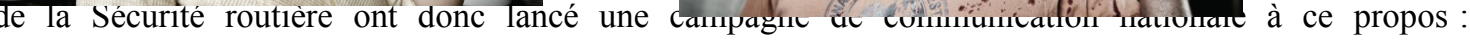

Téléphoner en conduisant multiplie par cinq les risques d'accident.

Des campagnes publicitaires de communication sociale similaires à celles-ci sur la programmation du GPS, ipod et d'autres dispositifs électroniques... ne cessent de se succéder sans doute pour nous faire prendre conscience du respect exigé lors de nos comportements sociaux. Dès les premières études sur les accidents de la route il s'est avéré que le comportement humain est la cause essentielle de l'insécurité routière (manque d'attention, défaut de maîtrise du véhicule...). Dans les dernières années, la sécurité routière s'apparente à une véritable propagande dirigée par un unique mode de pensée, celui de la répression, de la menace. Cependant, les statistiques mettent en évidence l'inefficacité de ces approches : le résultat est juste à l'opposé de ce que l'on attendait au départ. C'est la pédagogie qui manque : il faudrait sensibiliser le conducteur à la réalité physique d'un véhicule en déplacement, à sa responsabilité sur la sécurité routière et lui donner les moyens et les stratégies pour apprendre à circuler en toute sécurité.

2. La violence implicite de certaines images a autant d'impact que la violence explicite. Prenons comme exemple la campagne lancée en 2010 par l'association des Droits des non fumeurs (DNF) pour lutter contre le tabagisme chez les jeunes (public cible) qui compare l'esclavage de la cigarette à l'esclavage sexuel. 

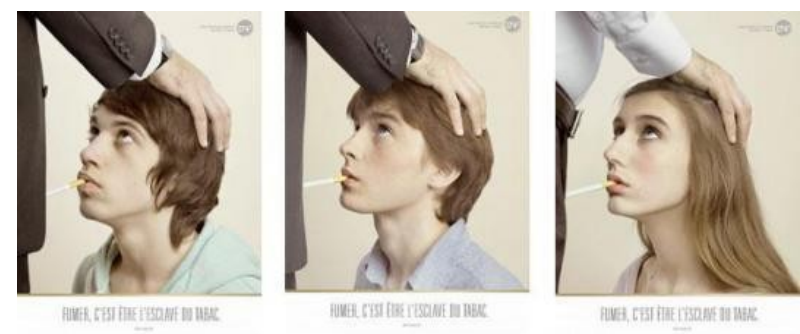

Pour accompagner la métaphore, un slogan : Fumer c'est être l'esclave du tabac. D'après le concepteur (Marco de la Fuente, vice-président de l'agence BDDP) de cette campagne polémique, cette publicité a pour but de mette en évidence la notion de soumission au tabac. Avec la cigarette, on s'impose la pire des soumissions, le pire des esclavagismes. On a cherché l'image de choc la plus emblématique de cette soumission : la soumission à la nicotine qui sert à rappeler la soumission sexuelle. Mais même si la campagne se veut explicite, on n'a pas eu l'intention de comparer ces deux registres (sexe et tabac) mais de communiquer avec les jeunes qui n'entendent pas le discours sanitaire et moralisateur. La sexualité étant chez eux une question très importante, on l'a considérée comme l'instrument le plus facile pour leur faire passer ce message.

3. La saillance liée à des inférences entre ce qui est connu et ce qui est nouveau, entre posé et présupposé (Grice, 1975). Cette question polémique a donné lieu à des interprétations diverses. Dans le domaine de la publicité sociale, le connu est saillant parce qu'il est cognitivement activé et parce qu'il est dans une certaine mesure stabilisé ; le nouveau est saillant parce qu'il est susceptible d'attiser l'intérêt, de focaliser notre attention.

4. La fréquente apparition des personnes domine le composant inanimé. Leur place sur l'image modifie le sens du message. Comme montre Pattabhiraman (1993: 40) le trait « humain » ou « animé » augmente la saillance de l'entité correspondante. On pourrait même établir entre elles une hiérarchie de saillance avant même de considérer la nature de l'événement qui les lie. Dans cette même optique s'inscrit la classification de Lyons (1980 : 142) entre personnes, animaux et objets inanimés; ceux-ci occupant le dernier échelon de cette hiérarchie.

5. Renforcement de l'impression de réalité par le fréquent emploi de la photographie. Réalité ? hyperréalité ? ou plutôt valeur hyperbolique des images spectaculaires qui y sont évoquées ? Les affiches publicitaires saisissent fréquemment des scènes réelles isolées de tout contexte, évoquant le côté le plus dur de la vie quotidienne. L'irruption violente et incisive des scènes ne laisse jamais indifférent le lecteur qui, touché dans ses sentiments les plus intimes, se sent dans l'obligation de se positionner par rapport au message transmis.

6. La « polysémie » ou « polyvalence» de certaines images. Le sens qui est attribué à chaque image est orienté par une mise en relief des images qui l'environnent, la légende qui l'accompagne et en fonction de son cadrage. Le recadrage de l'image permet d'orienter rapidement l'œil vers un point fort de celle-ci imposant une lecture dominante (techniques cinématographiques). De là l'importance du contexte comme effet de saillance. Montrer ou supprimer le contexte permet d'orienter l'intérêt du récepteur. Une image plein cadre décontextuée aura, en plus d'un rôle d'attestation, une fonction de valorisation de la personne représentée, de l'événement évoqué... Si le choix se porte sur un cadrage en gros plan, ce seront la personnalité, le caractère, les caractéristiques qui prédomineront. La mise en œuvre de ces techniques permet également si nécessaire le renforcement d'une image par une autre image ou le contraire, contamination et dévalorisation dépendant du choix effectué. Pensons à l'image d'une famille qui se 
dispose à prendre sa voiture pour partir en vacances. Cette image ayant pour scénario la scène d'un accident de la route à conséquences catastrophiques, le message est : «Précaution au volant lors de grands départs » (circulation routière). Si par contre, la scène portant sur la même famille avait comme fond de référence un paysage paradisiaque le message serait tout à fait divers : «Profitez des vos vacances dans un endroit exceptionnel ». Cet effet, inventé par le cinéaste soviétique Koulechov (né en 1899) repose sur le principe selon lequel l'esprit lie les images entre elles afin de leur donner un sens. Une même image donne lieu à des interprétations différentes par la mise en évidence des aspects psychologiques ou socioculturels souhaités.

7. La priorité esthétique accordée ou non au message visuel implique la direction ou l'indirection de la référence au produit, motivant par ce fait la participation active du récepteur qui s'attache au déchiffrage d'un message par lequel il se sent tout particulièrement touché. Bien encadrée dans un contexte spaciotemporel précis grâce aux ressources iconiques, l'approche publicitaire devient un acte socioculturel. S'appuyant sur les données matérielles de l'image (formats, codes formels, chromatiques...), l'argumentation iconique apparaît comme une occurrence proliférante susceptible de produire de multiples impressions sémantiques en fonction du public visé et du positionnement adopté par rapport au message transmis. L'opacité de certaines images se trouve canalisée par la mise en place des structures visuelles calculées en vue de provoquer des effets perceptifs concordants. Les effets produits par l'image se remarquent soit par leur prégnance soit par la redondance de leurs procédés soit par leur contraste avec leur entourage.

Les schémas iconiques fonctionnent comme supports visuels des concepts projetés par l'image susceptibles d'influencer leur réception. Ces concepts constituent dans la plupart des cas des unités argumentatives assez stéréotypées, fondées sur les croyances communes, sur une idéologie implicite qui entourent la pratique publicitaire, sur des représentations liées à une société et à une culture précise.

Dans le but de transmettre ces concepts le concepteur d'une image a deux options : soit diffuser un concept en différentes images, soit superposer différents concepts sur une même image accentuant par ce fait sa densité sémantique et augmentant son rendement. Dès cette perspective, l'image publicitaire devient un vrai filtre argumentatif chargé de la transmission d'instructions que le lecteur peut ou non réactiver.

\subsection{Saillance et symboles intentionnels}

La communication passe par les mots mais aussi par les sons, les formes, les couleurs. Dans une annonce publicitaire la langue porte en elle une musicalité, une charge de sens et d'émotion : Sans ceinture c'est tabou, on en viendra tout à bout. Tout en faisant parfois fi de la règle du mètre constant, le slogan peut se régulariser autour d'un centre: Confiance d'accord, mais prudence d'abord (SIDA). On peut même surdéterminer l'un des éléments de la prosodie : Prévenir vaut mieux que mourir ou présenter un écart par rapport à la norme : Protège-toi et protège les autres. La structure anaphorique de ces deux phrases révèle un procédé microstructural qui met en exergue la pluralité des sens d'une même signification. Les ressources symboliques de mise en relief employées sont nombreuses mais en tout cas, l'exigence créatrice doit être triple puisque en plus du visuel et du textuel il faut tenir compte de la sonorité du slogan publicitaire ou de la légende sous-jacente (registre émotionnel interpellé, interprétation du texte, structure de la phrase, choix des mots et des sons...).

Les sonorités positives, euphoniques contrastent avec des sonorités négatives ou déplaisantes. Il y a également des sons expressifs et des sons impressifs qui dans le domaine du psychisme renforcent l'impact d'un slogan ou d'un certain mot insistant sur la cohérence nécessaire entre texte et musicalité.

$\mathrm{Au}$ niveau de l'intonation le premier facteur de saillance est l'accent de focalisation ; le deuxième, l'intonation montante et en général, tout effet prosodique inhabituel et intentionnel. Pourtant, le pouvoir du rythme est fréquemment lié au pouvoir évocateur des couleurs et des formes. Le choix effectué au sein du code chromatique permet de mettre en relief un message, par ex. l'image d'un feu rouge en noir et blanc taché de sang (rouge, danger ; brûler un feu rouge peut entraîner de graves conséquences); le bleu 
par contre, dynamise les teintes chaudes ; quant au noir, il est susceptible d'exprimer soit des symboles positifs (solennité, distinction) soit des symboles négatifs (mort, solitude, peur). Procédures inductives et déductives jouant sur l'association, l'analogie et autres, constituent la base de l'argumentation iconique et des effets de saillance.

Par rapport aux formes, la mise en relief doit s'appuyer sur une symétrie " cachée », fondée sur la juxtaposition des contraires, sur la rupture du quotidien. La morphologie de l'image rend souvent l'argumentation iconique beaucoup plus impressive que ne l'est l'argumentation textuelle.

L'image cohérente, suggestive est fortement implicative dans ses effets. Le statisme des images contraste avec la vitesse qui marque la succession des événements ; leur éventuelle simplicité met en évidence les graves conséquences des événements invoqués.

Les caractères typographiques (différence de caractères, taille, graisse...) permettent de classer l'information selon une échelle hiérarchique ou en fonction du sentiment que l'on veut tout particulièrement exalter.

Finalement, une petite évocation des valeurs symboliques des gestes, des décors, des objets s'impose : deux images peuvent communiquer des perceptions contraires d'un même événement selon le trait le plus saillant : le visage grave ou souriant des personnes qui y figurent, la présence ou absence d'une marque de menace, de violence ou au contraire, d'affection, d'accueil.

\section{Conclusion}

Le langage en publicité sociale s'insère dans un discours mixte dans lequel différents codes fusionnés coexistent (langagiers, iconiques, verbaux...). Ces codes s'inscrivent dans une dynamique langagière qui, par la mise en évidence des aspects tout particulièrement déroutants, fait naître une prise de conscience collective sur les thèmes abordés.

Des paramètres linguistiques (verbaux et para-verbaux) et sémiotiques en interaction constituent les points forts sur lesquels repose la notion de saillance dans le domaine de la publicité sociale. La multicanalité du langage justifie d'une certaine façon la multiplicité des phénomènes de saillance et leur contribution, lorsqu'ils sont appliqués à des situations concrètes, à la sécurisation et à la valorisation sociales.

Ce «langage silencieux» dont parle l'anthropologue E.T. Hall (1973) est en réalité, dans la plupart des cas, « un appel au secours » d'une société désorientée à la recherche des valeurs authentiques.

Les exemples analysés dans le développement de notre étude montrent bien comment dans le domaine publicitaire l'interprétation d'un message est basée sur la saillance préalable des entités discursives et des objets perceptibles dans une situation de communication, mais la production du langage fait également intervenir la saillance, une «saillance nouvelle» selon la finalité communicative recherchée.

\section{Références bibliographiques}

Act Up-Paris : http://www.actupparis.org/spip.php?article3022 [06/09/2011]. 
Adam, J. M. (1975). «Enjeux d'une approche du discours publicitaire: notes pour un travail de recherche», Pratiques, $\mathrm{n}^{\circ}$ 7/8, Metz, 23-35.

Adam, J. M. et Bonhomme, M. (1997). L'argumentation publicitaire. Paris : Armand Colin, coll. « Cursus ».

Association Prévention $\quad$ Routière. $\quad$ http://www.preventionroutiere.asso.fr/shared/cmd.aspx?
domain=ugo\&action=pagelet [07/09/2011].

Austin, J. L. (1962, 1970). Quand dire c'est faire. Paris : Seuil.

Barthes, R. (1964). « Rhétorique de l'image ». Communications, n 16. Paris : Seuil, 78-92.

Baylon, C., Mignot, X. (1994, 1999). La communication, 2ème éd. Paris : Nathan Université, coll. «fac linguistique ».

Beacco, J.-C., Moirand, S. (1995). Les enjeux des discours spécialisés. Paris : Presses de la Sorbonne Nouvelle, Les carnets du Cediscor 3

Blum, Y. et Brisson, J. (1971). « Implication et publicité », Langue française, $\mathrm{n}^{\circ}$ 12. Paris : Larousse, 46-61.

Cadet, C., Charles, R. et J.-L. Galus. (1990). La communication par l'image. Paris: Nathan, coll. « repères pratiques ».

Declercq, G. (1992). L'art d'argumenter. Paris : Éditions Universitaires.

Les Échos : http://www.blogs.lesechos.fr/article.php?id_article= 3173 [08/09/2011].

Eco, U. (1988). Sémiotique et philosophie du langage. Paris : PUF.

Everaert-Desmedt, M. (1984). La Communication publicitaire. Étude sémio-pragmatique. Louvain-la-Neuve : Cavay.

Gouvernement français : http://www.securite-routiere.equipement.gouv.fr [08/09/2011].

Grize, H. P. (1975). « Logique et conversation ». Communications, nº 30. Paris : Seuil, 45-51.

Gruning, B.-N. (1990). Les mots de la publicité. Paris : Presses du CNRS.

Hall, E. T. (1973). Le langage silencieux. Paris : Édition du Seuil, coll. « Points ».

Jakobson, R. (1963). Essais de linguistique générale. Paris : Éditions de Minuit.

Jakobson, R. et Waugh, L. (1980). La charpente phonique du langage. Paris : Éditions de Minuit.

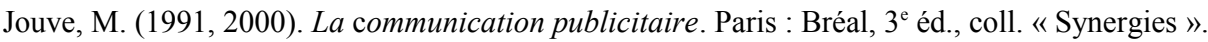

Khramer, E. \& Theune, M. (2002). «Efficient context-sensitive generation of referring expressions ». Information Sharing : Givenness and Newness in Language Processing, K. van Deemter and R. Kibble (eds.). Stanford : CSLI Publications, 223-264.

Landragin, F. (2004). Saillance physique et saillance cognitive. Cognition, Représentation, Langage (CORELA) 2 (2), http://edel.univ-poitiers.fr/corela [06/09/11].

Lits, M. (2008). Du récit au récit médiatique. Bruxelles : De boeck. 
Lyons, J. (1980). Sémantique linguistique. Paris : Larousse.

Le Parisien : http://www.lepost.fr/sondage/2010/02/23/1954967 campagne-choc-anti-tabac-est-elle [24/10/2010].

Pattabhirama, T., Cercone, N. (1990). « Selection : Saliance, Relevance and the Coupling beteween Domain-Level

Tasks and Text planning ». Proceeding of the Fifth International Workshop on Natural Language Generation, Dawson, 79-86.

Pereleman, C. et Olbrechts-Tyteca, L. (1958, 2008) (5éd.). Traité de l'argumentation. Bruxelles : Éditions de l'université libre de Bruxelles.

Rastier, F. (1991). Sémantique et recherches cognitives. Paris : Presses Universitaires de France.

Rastier, F., Cavazza, M. et Abeillé, A. (1994). Sémantique pour l'analyse. Paris : Masson.

Robert, P. (2004). Le Nouveau Petit Robert. Paris : Le Robert.

Rousselet, G. A., Fabre-Thorpe, M. (2003). « Les mécanismes de l'attention visuelle ». Psychologie Française, 48, I, 29-44.

Speedy Z Corporation (The) : http://speedy-ovh.org/index.php?art [24/10/2011].

Stevenson, R. J. (2002). "The Role of Salience in the Production of Referring Expressions». Van Deemter, K., Kibble, R. (eds.). Information Sharing : Reference and Presupposition in Language Generation and Interpretation. Stanford : CSLI Publication, 167-192.

Victorri, B. et Fuchs, C. (1996). La polysémie. Construction du sens. Paris : Hermes.

Votre santé au quotidien (magazine): http://www.e-sante.fr/accidents-C 1-6 [08/09/2011].

Wolters, M. K. (2001). Towards Entity Status. Ph. D. Thesis Bon University.

http://desyeuxpourcomprendre.spot.com/leffet-koulechov.html [04/11/2011]. 